\title{
A scalable approach for future vehicle electrification
}

Dr. Carsten Bünder

Director Product Management, Magna Powertrain, Transmission Systems

This manuscript is not available according to publishing restriction.

Thank you for your understanding. 